\title{
Computer Aided Diagnosis System for Lung Cancer Based on Helical CT Images
}

\author{
Y.Kawata ${ }^{1}$, K. Kanazawa ${ }^{1}$ S. Toshioka ${ }^{1}$, N.Niki ${ }^{1}$, \\ H. Satoh ${ }^{2}$, H. Ohmatsu ${ }^{3}$, K. Eguchi ${ }^{4}$, N. Moriyam ${ }^{3}$ \\ ${ }^{1}$ Dept. of Optical. Science, Univ. of Tokushim a, Japan \\ ${ }^{2}$ Medical Engineering Laboratory, Toshiba Corporation, Japan \\ ${ }^{3}$ National Cancer Center Hospital East, Japan \\ ${ }^{4}$ National Cancer Center Hospital, Japan
}

\begin{abstract}
A bstract. Lung cancer is known as one of the most difficult cancers to cure. In order to improve the recovery rate for lung cancer, detection and treatment at an early stage of growth is necessary. For the purpose, chest CT images obtained by helical CT scanner have drawn interest in the detection of suspicious regions. This paper present a method to improve the detection accuracy of the system that we have developed to detect candidates of lung cancer based on helical CT images. The basic improvement of the system is to enhance the difference of intensity surfaces between suspicious and normal regions by using surface curvatures such as the Gaussian and the mean curvatures. Experiments to show its feasibility of improving the detection accuracy are demonstrated by applying the method to the chest CT images.
\end{abstract}

\section{Introduction}

According to figures of cancer incidence in Japan mortality from cancer is increasing annually with the present rate for cancer prevailing in a quarter of all deaths. Among deaths from cancer, lung cancer is the most common cause, accounting for $21.4 \%$ of all cancer deaths for males in Japan[1]. In order to improve the recovery rate for lung cancer, detection and treatment at an early stage of growth is necessary. As a conventional method for the mass screening process, chest X-ray films have been used for lung cancer diagnosis. Since the chest X-ray files are twodimensional projection images, the overlapping of bone and organs shadows results in disturbing the detection of small lung cancers at early stage.

Recently, chest CT images obtained by helical CT scanner which can make a wide range measurement of lung in a short time have drawn interest in detection of suspicious regions[2]-[7]. However, mass screening based on helical CT images leads to a considerable number of images to be diagnosed. This time-consuming fact makes it difficult to be used in the clinic. To increase the efficiency of the mass screening 
process, we are working towards a computer-aided diagnosis system for lung cancer detection based on helical CT images. A prototype computer aided diagnosis (CAD) system to automatically detect suspicious regions from chest CT images and alert physicians attention has been built by our group $[5,6]$. The key ingredients of the system are the image analysis and the image diagnosis parts. The first part consists of the following procedures; the extraction of lung area, the correction of partial volume effect and beam hardening, the extraction of region of interest (ROI), and the feature extraction from ROI. The second part detects suspicious region and alerts physicians attention by applying diagnosis rules acquired from physicians. The experimental results of the prototype system by using 450 cases which have been diagnosed by three physicians presented good performance except for eleven false negative cases which were difficult cases to be diagnose by even physicians[6]. Since these false negative cases have small size (less than $5 \mathrm{~mm}$ diameter) and faint gray level, their shape features measured by the image analysis part are not enough to discriminate suspicious regions from normal regions. In order to improve the detection accuracy it is desired to develop a method to enhance the slightly difference between suspicious and normal shade regions.

In this paper, we concentrate on the application of surface curvatures on intensity surface of chest CT slices images to extract slightly different characteristics between suspicious and normal regions. This paper is organized as follows. In the next section, an overview of the prototype CAD system and its performance are presented. Then, in Section 3, a modified method is given to improve the detection accuracy of the CAD system. The Section 4 demonstrates experimental results of the modified method using the difficult cases for the original system. Section 5 present our conclusion concerning our developed CAD system.

\section{System Overview}

\subsection{Image Analysis Part}

The prototype CAD system to detect the candidates of lung cancer from helical CT images consists of two parts; image analysis part and image diagnosis part. The input to this system are helical CT images acquired under the following conditions; beam width: $10 \mathrm{~mm}$, table speed: $20 \mathrm{~mm} / \mathrm{sec}$, tube voltage: $120 \mathrm{kv}$, tube current: $50 \mathrm{~mA}$. For the scan duration(about $15 \mathrm{sec}$ ), patients held their breath at full inspiration. 35 slices per patient at $10 \mathrm{~mm}$ intervals are obtained. Pixel size in each slice is $0.6 \mathrm{~mm} \times 0.6 \mathrm{~mm}(512$ pixel matrix).

The first part of the system deals with the extraction of lung area, the correction of the partial volume and the beam hardening effects, the extraction of region of interest $(\mathrm{ROI})$, and finally the feature extraction of ROI $[5,6]$. These procedures are presented briefly.

Step 1) Extraction of lung area: The lung area are mostly occupied by air, so its CT 
values are low within the lung area. The extraction process of the lung area is based on a thresholding algorithm. However, this thresholding technique excludes the lung boundary with high CT values from the real lung area. In order to avoid this problem the lost parts are compensated by using the curvature at the boundary of the initial lung area's contour and the position of the rib regions extracted from the same CT slices[5,6].

Step 2) Correction of the partial volume and the beam hardening effects: The helical $\mathrm{CT}$ images involve artifacts by the beam hardening effect and the partial volume effect. These artifacts which affect the ROI extraction procedure are corrected by a simple technique used to smooth the lung area image based on mathematical morphology operation.Then, the smoothed images are subtracted from the original lung area image[5,6].

Step 3) Extraction of ROI: The interesting organs in the lung, such as blood vessels and tumors, are segmented within the lung area obtained by the above step. The lung area images are segmented into two clusters, air cluster and other organs cluster by using fuzzy clustering method[8,9]. The small size regions contained in the organs clusters are eliminated by the gray-weighted distance transformation technique[10].

Step 4) ROI Feature extraction: This step extracts the shape features of each ROI. The following features are considered; area, thickness, circularity, gray level, variance of gray level, position in the lung area. The detail calculations of these features are described in $[5,6]$.

\subsection{Image Diagnosis Part}

The second part of the prototype system detects suspicious region and alerts physicians attention by applying diagnosis rules acquired from physicians. Three diagnosis rules are constructed based on the following diagnostic medical knowledge;

1 ) The shape of the lung cancer is generally spherical, and it is seen like a circle on the $2 \mathrm{D}$ cross section. However, the shape of the blood vessels running horizontally is oblong,

2 ) The thickness of the blood vessel becomes smaller as its position is near the lung wall, however the thickness of the lung cancer is generally larger than the ordinary thickness of the blood vessels elsewhere,

3 ) The shadows contacting the lung wall are generally tumors, because the periphery blood vessels are too small to be seen in the helical CT image and are difficult to be recognized,

4 ) The pixel values of the blood vessels are generally higher than the lung cancer when running vertically in a helical $\mathrm{CT}$ image using the measurement conditions mentioned previously, 
Table I Criterion of judgment

\begin{tabular}{|c|c|}
\hline Judgment & lesion type \\
\hline E & sure malignant lesion \\
\hline D & probably malignant lesion \\
\hline C & non malignant lesion \\
\hline B & normal case \\
\hline
\end{tabular}

Table II Comparative results between physicians and the prototype CAD system

\begin{tabular}{|c|c|c|c|}
\hline Judgment & $\begin{array}{c}\text { The number of suspicious } \\
\text { regions by physicians }\end{array}$ & $\begin{array}{c}\text { The number of suspicious } \\
\text { regions by CAD system }\end{array}$ & False negative \\
\hline E & 11 & 11 & 0 \\
\hline D & 109 & 98 & 11 \\
\hline total & 120 & 109 & 11 \\
\hline
\end{tabular}

5 ) The pixel values of the lung cancer regions are comparatively uniformed. The diagnosis rules are classified into the following three types;

Rule 1: This rule eliminates the ROI which is not obviously suspicious, because most of the extracted ROI are blood vessels regions.

Rule 2 : This rule detects the suspicious regions without contacting the lung wall.

Rule 3: This rule detects the tumors contacting the lung wall. The implementation of these rules are detailed in $[5,6]$.

\subsection{Performance Evaluation}

To evaluate the performance of the prototype CAD system, the system was applied to the helical CT images of 450 cases (total: 15,750 slice images)[6]. All data are diagnosed by three physicians with the criterion shown in Table $I$. The malignant cases including the lung cancer and other lung lesion are discriminated from the benign cases by agreement of three physicians diagnoses. Table II shows the comparative results between the physicians and the prototype system. In this results all the judgment $\mathrm{E}$ type lesions are detected perfectly by the CAD system. The prototype CAD system successfully detects suspicious regions from the chest CT images and could be very useful in assisting lung cancer screening. However some limitations are notified in that as shown in Table II. There are eleven false negative cases which are difficult to be diagnosed by even physicians. Since these cases size is smaller than $5 \mathrm{~mm}$ diameter and the gray level in these suspicious region is occupied 
by low contrast, it is difficult to extract shape features through the image analysis part of the prototype CAD system. Especially, the correction step of the artifacts and the extraction step of ROI in the image analysis part have tendency to lose the suspicious regions with small size and low contrast.

\section{Improvement of Image Analysis Part}

This section introduces a method to reduce the number of false negative case mentioned in Section 2.3. The advantage of this method is that the extraction of ROI from the lung area images is performed by surface curvatures without correction of the artifacts such as the partial volume and the beam hardening effects. To remove the correction of these artifacts, we replace Step 2 and Step 3 mentioned in Section 2.1 with the following two steps, Step 2' and Step 3'.

Step 2) Calculation of surface curvatures : This step computes the Gaussian and the mean curvatures on the intensity surface of the lung area image. The curvature calculation method is as follows; first we perform the convolution with the Gaussian function to transform the digital 2D lung area image into an infinitely differentiable 2D function $\mathrm{I}(\mathrm{x}, \mathrm{y})$; then, get the the Gaussian curvature $(\mathrm{K})$ and the mean curvature $(\mathrm{H})$ expressed in Eqs.(1) and (2) [12].

$$
\begin{aligned}
& K=\frac{1}{d}\left(I_{x x} I_{y y}-I_{x y}^{2}\right) \\
& H=\frac{1}{2 d^{3 / 2}}\left(I_{x x}-2 I_{x} I_{x y} I_{y}+I_{x x} I_{y}^{2}+I_{y y}+I_{y y} I_{x}^{2}\right) \\
& d=1+I_{x}^{2}+I_{y}^{2}
\end{aligned}
$$

where the subscripts denote the partial differentiation.

Step 3') Extraction of ROI: The ROI which tend to be the convex and hyperbolic surface regions on the intensity surfaces. To extract convex and hyperbolic surface regions the calculated curvatures signs are utilized. The signs of these surface curvatures segment the intensity surface into eight basic viewpoint-independent surface types [11], such as peak surface $(K>0$ and $H<0)$, flat surface $(K=0$ and $H=0)$, pit surface $(K>0$ and $H>0)$, minimal surface $(\mathrm{K}<0$ and $\mathrm{H}=0)$, ridge surface $(\mathrm{K}=0$ and $\mathrm{H}<0)$, saddle ridge $(\mathrm{K}<0$ and $H<0$ ), valley surface $(K=0$ and $H>0)$, and saddle valley ( $K<0$ and $H>0$ ). Since it is difficult to decide whether $\mathrm{K}$ and $\mathrm{H}$ are zero or not[13], the four surface types including flat surface, minimal surface, ridge surface, and 
valley surface are not assigned in practical experiments. In this step, we consider the following surface types regions as ROI; pit surface, saddle ridge, and saddle valley surface types. The small size regions contained in the organs clusters are eliminated by the gray-weighted distance transformation technique[10].

\section{Experimental Results}

In order to demonstrate the feasibility of the modified method for improving the detection accuracy, we applied the modified method to the difficult cases for the prototype $C A D$ system. In this experiment the image diagnosis part utilized the same as the prototype CAD system.

Fig. 1 shows the application result of the modified method to one difficult case of the eleven false negative cases of the prototype CAD system. Fig.1(a) is original chest $\mathrm{CT}$ image and an arrow denotes the candidate of lung cancer specified by the physicians. Fig.1 (b) shows the bird's-eye view of the intensity surface of the lung aria extracted from the slice image in Fig.1(a). Fig.1 (c), (d), and (e) present the surface segmentation result by using surface curvatures, the extraction result of ROI, and the detection result of suspicious regions, respectively. The defined color-code corresponding to each surface type is as follows; peak surface is white, pit surface is green, saddle ridge is blue, and saddle valley is red. In Fig.1(c), the color-code distinguishes four surface types; peak surface, saddle ridge, saddle valley, and pit surface. In Fig.1(d), the black regions present the extraction result of ROI from the lung area image. In Fig.1(e) the detected position is superimposed on the original chest $\mathrm{CT}$ image with red color-code. The small suspicious regions with low contrast are difficult to be detected through the original image analysis part. However, with more precise diagnostic rules, the modified method seems promising good efficiency to extract the small suspicious regions as ROI. In that, actually it has reduced the eleven false negative cases diagnosed by the previous method to seven false negative cases.

\section{Conclusion}

We have suggested a method to reduce the number of false negative cases caused by the prototype CAD system for lung cancer under development. The method is based on surface curvatures such as the Gaussian and the mean curvatures on the intensity surface of the helical CT images. Experimental results with chest CT images including difficult diagnosis cases of lung cancers, such as low contrast regions, demonstrated the feasibility of our method to suppress the number of false negative cases. Our future aim is to develop image diagnosis rules to deal with the image features obtained by the method. 


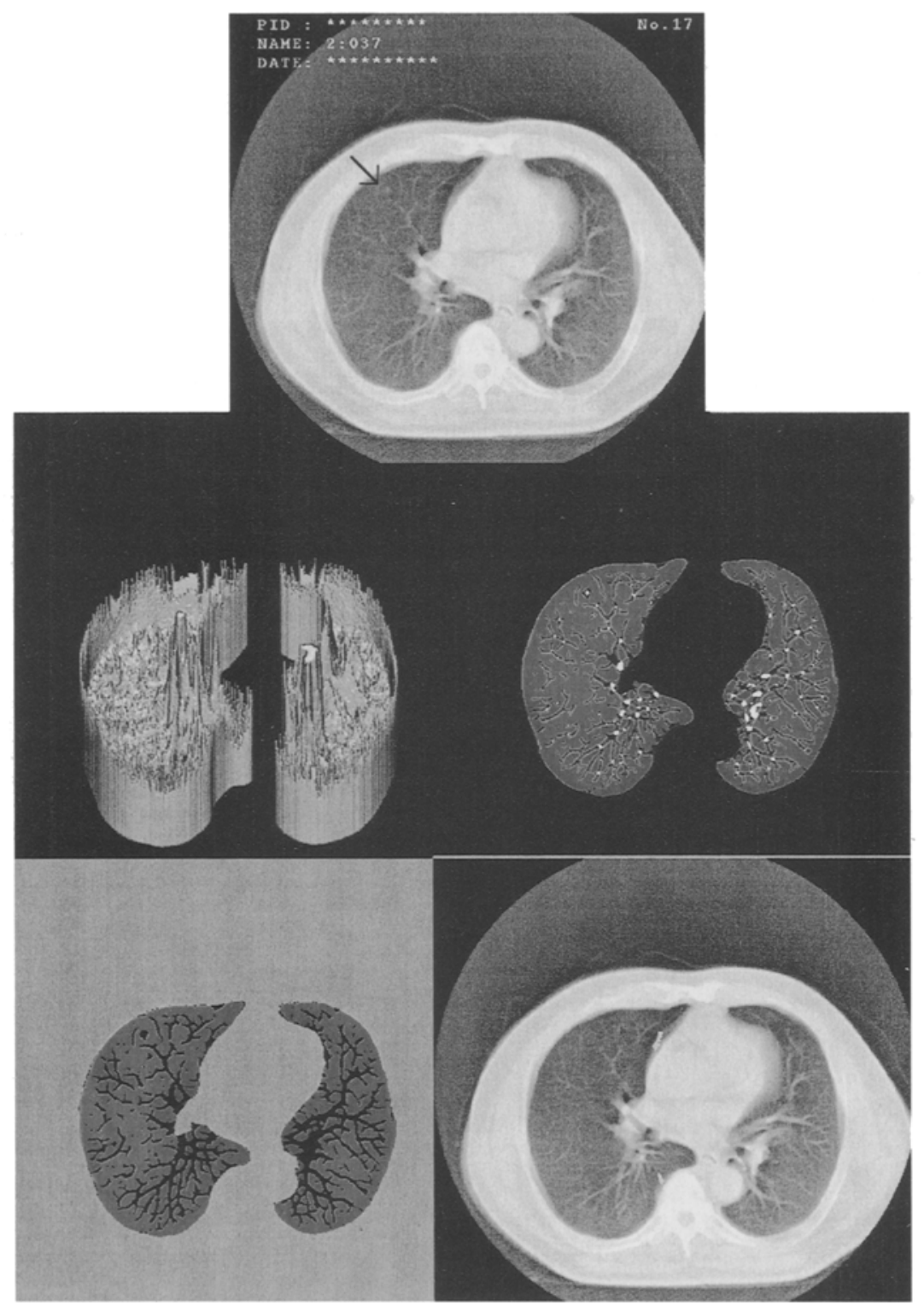

Fig. 1 Application result of the improved method. (a) Original chest CT image. The arrow denotes the candidate of lung cancer specified by the physicians. (b) Bird'seye view of the intensity surface of the lung area. (c) Surface segmentation result by using surface curvatures. (d) Extraction result of ROI. (e)Detection result of suspicious regions. The defined color-code corresponding to each surface type; peak surface : white, pit surface : green, saddle ridge : blue, and saddle valley : red. 


\section{References}

1. T.Kaizoe et al. Eds.: Figures on cancer in Japan-1995, Foundation for Promotion of Cancer Research, Japan, 1995.

2. N.Moriyama, R.Iwata, F. Wako, M.Ohtani and H.Ohmatsu: Helical computed tomography scanning of the thorax and abdomen, Jpn. J. Clin. Oncol., vol.23, pp.156-161, 1993.

3. M.Kaneko, K.Eguchi, H.Hirano, H. Ohmatsu, and the Anti-Lung Cancer Association(ALCA) Tokyo: Chest helical CT (CHCT): a utility in the early detection of peripheral lung cancer (PLC), Am. J. Resp. Critical Care Med., vol.154, p.A772, 1995.

4. T.Iinuma, Y.Tateno, T.Matsumoto, S.Yamamoto, M.Matsumoto: Preliminary Specification of X-ray CT for Lung Cancer Screening (LSCT) and its Evaluation on Risk-Cost-Effectiveness, Nippon Acta Radiologica, Japan, vol.52, no.2, pp.182-190, 1992.

5. K.Kanazawa, N.Niki, H.Nishitani, H.Satoh, H.Omatsu, N.Moriyama: Computer Assisted Diagnosis of Lung Cancer Using Helical X-ray CT, IEEE Computer Society Press, pp.261-267,1994.

6. K.Kanazawa, K.Kubo, N.Niki, H.Satoh, H.Ohmatsu, K.Eguchi, N.Moriyama: :Computer Aided Screening System for Lung Cancer Based on Helical CT Images, Visualization in Biomedical Computing, Lecture Notes in Computer Science, Springer, pp.223-228, 1996.

7. M.L.Giger, K.T.Bae, $\mathrm{H}$ and MacMahon: Computerized Detection of Pulmonary Nodules in computed Tomography Images",Invest Radiol,vol.29,no.4,pp.459$465,1994$.

8. M.M.Trivede, J.C.Bezdek: Low-Level segmentation of aerial images with Fuzzy clustering, IEEE Trans.Syst., Man. \& Cybern., SM C-16, 4, pp.589-59, 1986.

9. N.Niki, Y.Kawata, H.Satoh: A 3-D Display Method of Fuzzy Shapes Obtained from Medical Images, Trans.IEICE, Japan, vol.J73-D-II,no.10,pp.1707-1715, 1990.

10. J.Toriwaki, A.Fukumura, T.Maruse: Fundamental Properties of the Gray Weighted Distance Transformation, Trans.IEICE, Japan, vol.J60-D, no.12, pp.1101-1108, 1977.

11. P.J. Besel and R.C. Jain: Invariant surface characteristics for 3D object recognition in range images, Comput. Vision, Graphics, and Image Processing, vol.33, pp.33-80, 1986.

12. J.-P. Thirion and A. Gourdon: Computing the differential characteristics of isointensity surfaces, Comput. Vision and Image Understanding, vol.61, pp.190-202, 1995.

13. R. Jain, T. Sripradisvarakul, and N.O.Brien, :Symbolic surface descriptors for 3dimensional object recognition, in Proc. SPIE, vol.754, pp.82-91, 1987. 